\title{
A test of time sharing in auditory attention
}

\author{
WILLIAM P. BANKS and JEFFREY P. ZENDER \\ Pomona College, Claremont, California
}

\begin{abstract}
This study shows that subjects, while shadowing a primary message, can monitor two additional messages as well as they can monitor a single one. In addition, monitoring for targets on the primary message is not impaired more by two than by one additional message to monitor, and performance on all monitoring tasks appears to approach an asymptote of no interference. These findings contradict any limited-capacity theory of attention that attempts to use speeded time sharing of limited resources to explain the fact that subjects can perform two skilled tasks at once. If subjects were time sharing at any stage of practice, an increase in the number of alternative channels to be monitored would have made performance worse.
\end{abstract}

Several lines of investigation have, in recent years, challenged the limited-capacity hypothesis that has become an explicit and implicit assumption of informationprocessing research and theory (cf. Eriksen \& Spenser, 1969; Hirst, Spelke, Reaves, Charack, \& Neisser, 1980; Schneider \& Shiffrin, 1979; Spelke, Hirst, \& Neisser, 1976). This hypothesis takes various forms. One is the "'single-channel hypothesis," which asserts that skilled, attentionally directed processing must be accomplished by a single limited-capacity processor of somewhat flexible abilities. This processor is often the "bottleneck" in theories of attention, and in these theories it usually needs to be protected from overload by "filters" placed on channels leading to it. The processor has sometimes been identified with awareness or consciousness, thereby gaining some intuitive justification from the introspective observation that awareness is limited in the number of things it can encompass at any given time. Possibly because of its intuitive justification, the single-channel hypothesis is more widespread than is often recognized. Virtually every information-processing flowchart implicitly assumes this hypothesis by providing a bottleneck for information flow at some point in the model of processing it proposes.

The findings challenging the single-channel assumption have been of two kinds: those showing an influence on behavior of nonconscious processing at a semantic level (Lewis, 1970; MacKay, 1973; Marcel, 1983) and those showing that the essential evidence for capacity limitations may only be the result of testing at low levels of practice (Hirst et al., 1980; Spelke et al., 1976). The latter findings are the topic of this article. These findings have been used to support the hypothesis that several "channels" of processing can exist when practice is adequate and thus that there is no intrinsic single-channel limitation. However, they are open to explanation by the alternative hypothesis that, with extended practice, subjects simply learn to deploy their one processing channel more

The authors' mailing address is: Department of Psychology, Pomona College, Claremont, CA 91711. efficiently. This is termed the time-sharing hypothesis because it proposes that the increase in efficiency comes about by a rapid switching of the one processing channel from one input to the other. The efficient deployment of the one processing channel only gives the impression that the single-channel limitation has been overcome.

Hirst et al. (1980) attempted to disconfirm the timesharing hypothesis by showing that training of skill with redundant material transferred to less redundant material. We consider this a weak test of the time-sharing hypothesis because it tests only a form of time sharing in which errorless use of the single channel is accomplished by exploiting the redundancy of the message. This test does not exclude strategies in which redundancy is not used.

This article reports a different test of the time-sharing hypothesis. If time sharing mimics multiple or parallel channels by successively allocating a serial processor to the relevant inputs, then at any given stage of practice it must take longer as the number of inputs it serves increases. This test therefore compares detection of messages on one, two, and three channels while one channel is being shadowed. This study also examines the effect of practice. If subjects begin with a time-sharing strategy but switch to simultaneous processing, the results should show better monitoring performance with fewer channels at the beginning of practice but no difference later on. If multiple processing can be accomplished only by time sharing, then some difference in performance will be maintained throughout practice.

\section{METHOD}

\section{Subjects}

Two males, 21 years of age, served as part of a work-study assignment. One had served in a pilot study and continued for 17 daily sessions, whereas the other was forced to quit the study after 10 sessions because of a serious accident unrelated to this study.

\footnotetext{
Apparatus, Material, and Procedure

All material was taped and presented to subjects through headphones. The subjects always heard three voices: One was presented exclusively to the left ear, one to the right, and one to both ears. The latter voice sounded as though it emanated from the center of the head. The three
} 
voices were recorded by different individuals and contained different material (selections from the Los Angeles Times on the left and right and from the Britannia Micropaedia in the center). The subjects always shadowed the center channel.

Monitoring for targets defined the three conditions: Targets were to be detected either on the shadowed channel alone, on just one of the two lateralized channels in addition to the shadowed channel, or on all three channels. Before a trial, the subjects were informed of the channels to be monitored for targets. Targets were defined by category, and specific category members were never repeated. The subjects were instructed to tap a ruler on a desk in front of them whenever they detected a target in the specified channel(s). They were instructed to ignore targets that might appear in nonmonitored channels, but in fact none was placed there.

Five tapes were generated for experimental trials. Each contained 10 1-min-long segments. The first of these was a warm-up segment with targets only in the shadowed channel, and the remaining 9 consisted of 3 segments in each of the 3 conditions. Every segment had all three voices, reading approximately 150 words of text each.

The shadowed channel always contained 10 target words. In conditions in which the nonshadowed channels were to be monitored, 10 more target words were added. When only one nonshadowed channel was to be monitored, all 10 were placed there; when both were to be monitored, 5 were put in each. Targets were placed randomly in the passages with the constraints that no two occur at the same time and none fall in the first or last 8 words. The targets were written into the text at preselected locations before the passages were recorded. The readers practiced reading the inserted words smoothly in the text, even though the target word never made sense in the context in which it was placed.

In each experimental session, the subjects shadowed practice material (with no competing message) and then performed on one of the five tapes. Each subject had a different order of the five tapes on the first five experimental sessions and then repeated the order over the subsequent sessions, going through the 10 segments on each tape in the same order each time. The experimenter monitored shadowing and detection. The subjects were reminded to shadow accurately when they made errors (errors were rare).

\section{RESULTS AND DISCUSSION}

Figure 1 shows the results obtained with Subject 1, and Figure 2 shows the results with Subject 2 . These results show very clearly that monitoring performance improves with practice, that the interference between monitoring the shadowed and nonshadowed channels declines with practice, and most important, that there is no difference between monitoring one and two nonshadowed channels. This latter conclusion is based on two sorts of evidence: Detection of targets on the shadowed channel (open symbols at top of figures) does not decline from one to two alternative channels, and detection of targets on rejected

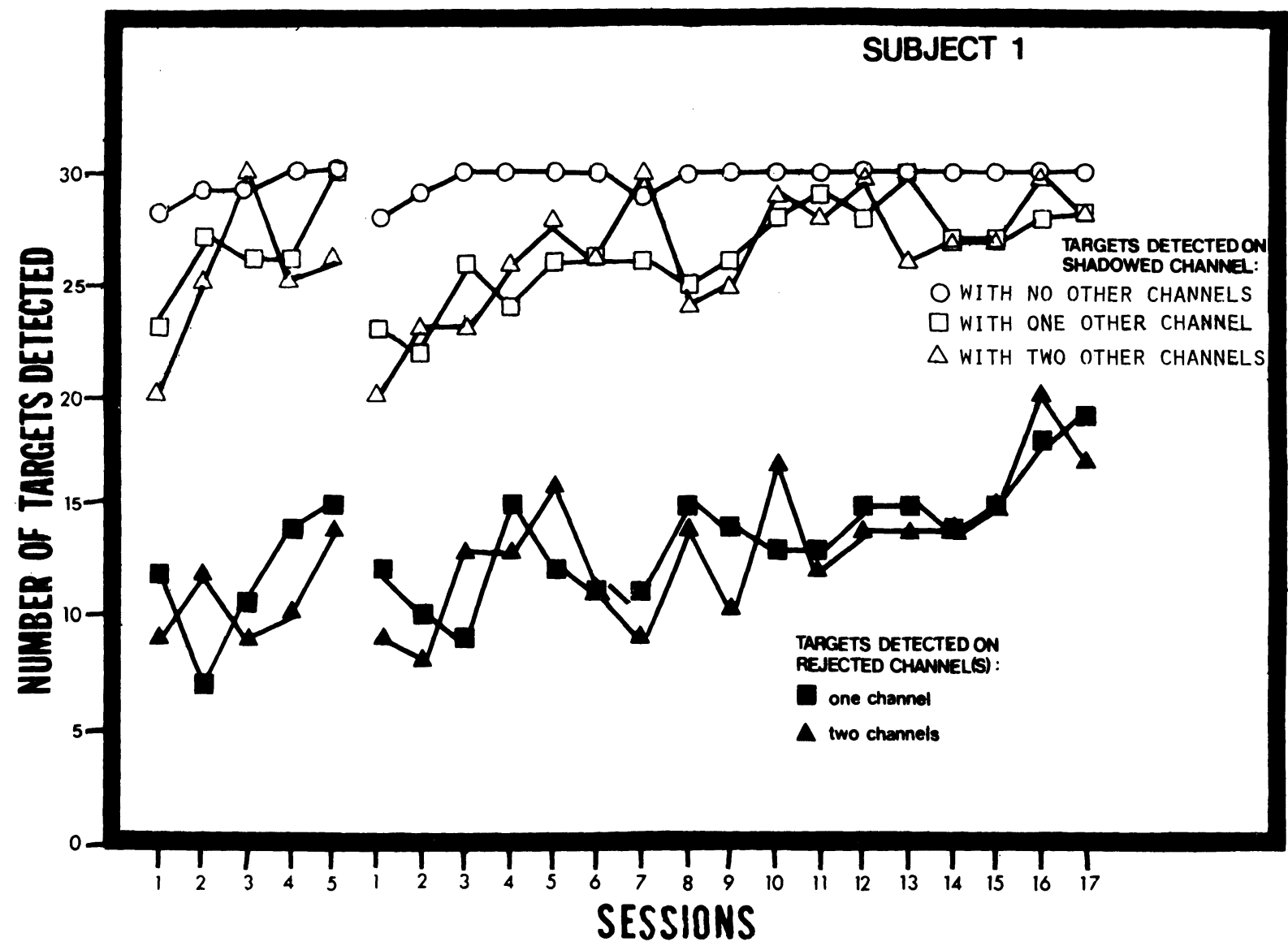

Figure 1. Detection of targets in three-segment units by Subject 1 on the shadowed channel (open symbols) and on nonshadowed channels (closed symbols). The circles plot detection with no alternative channels to monitor. The squares plot detection with one channel in addition to the shadowed channel to monitor and the triangles plot detection with two additional channels. The five trials plotted at the left are from practice sessions conducted with slightly different material and situation some weeks before the 17 experimental sessions plotted in the rest of the figure. 


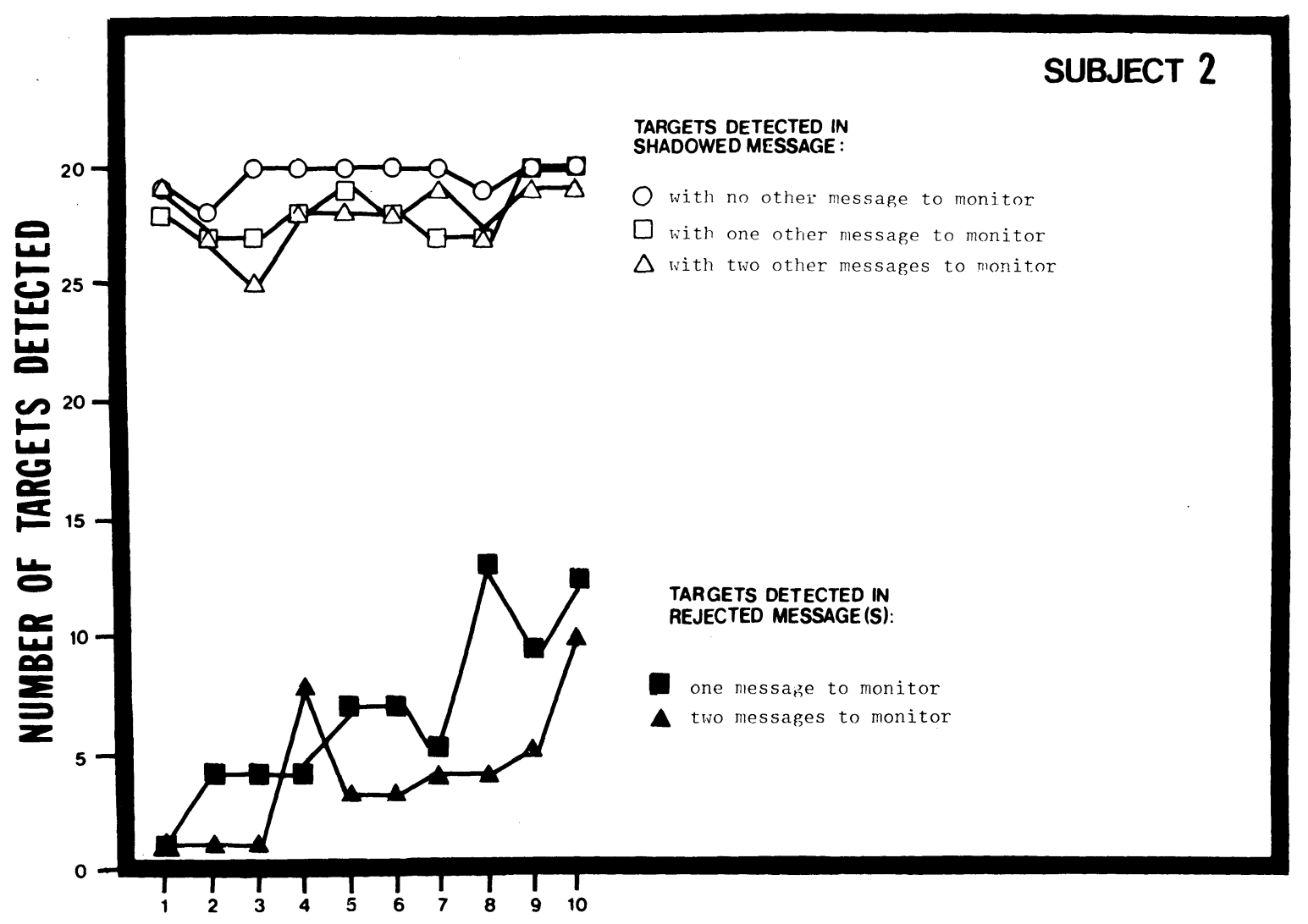

SESSIONS

Figure 2. Results for Subject 2, plotted according to the same conventions as Figure 1. Subject 2 did not have any practice sessions before the 10 experimental trials plotted here.

channels (closed symbols) also does not decline from one to two rejected channels. If the subjects were time sharing by alternating among input channels, the results would show a consistent advantage for monitoring one rather than two channels at each level of practice, whether the measure was detection of targets on the shadowed or on the nonshadowed channel. By this criterion, there appears to be some time sharing on the first two trials for Subject 1 and over the first three for Subject 2, but (with the exception of Trial 8 and possibly Trial 9 for Subject 2) no time sharing thereafter. (This result accords nicely with the reports of Hirst et al.'s (1980) subjects that they alternated attention between the tasks only at the beginning of practice.) It is clear that the lack of evidence for time sharing does not come from the lack of difficulty of the task; performance starts off at a very low level: under $30 \%$ correct detections for Subject 1 and under $15 \%$ for Subject 2. Furthermore, the improvement in performance over time shows that something was learned, but the near coincidence of the functions during learning indicates that the improvement did not come through acquiring more effective time-sharing strategies.

This experiment is, we note, quite similar to that of Treisman and Geffen (1967). It replicates their finding that targets on nonshadowed channels are not detected as well as targets on shadowed channels. However, it also shows that the difference between shadowed and nonshadowed channels declines with practice. In our experiment, subjects never overcame the difference between shadowed and nonshadowed channels, but it seems possible that with more practice there would be no interference between shadowing and monitoring:

In conclusion, we believe that we have provided the sort of strong support needed for Hirst et al.'s (1980) assertion that time sharing of a single processing channel cannot explain their results. We realize that it would be better to have results from more subjects, more sessions of practice, and more varied material. However, given the considerable technical difficulty of this research and the time required to obtain more data, we felt it best to publish these preliminary findings. These data (and limited data from other subjects not reported here) lead us to believe that further research will not force major changes in our conclusions.

\section{REFERENCES}

ERIKSEN, C. W., \& SPENSER, T. (1969). Rate of information processing in visual perception: Some results and methodological considerations. Journal of Experimental Psychology Monographs, 79(2, Pt. 2). 
Hirst, W., Spelke, E. S., Reaves, C. C., Charack, G., \& Neisser, U. (1980). Dividing attention without alternation or automaticity. Journal of Experimental Psychology: General, 109, 98-117.

LEWIS, J. L. (1970). Semantic processing of unattended messages under dichotic listening. Journal of Experimental Psychology, 85, 225-228.

MACKAY, D. G. (1973). Aspects of the theory of comprehension, memory, and attention. Quarterly Journal of Experimental Psychology, 25, 22-40.

MARCEL, A. J. (1983). Conscious and unconscious perception: Experiments on visual masking and word recognition. Cognitive Psychology, 15, 197-237.
Schneider, G. E., \& Shiffrin, R. M. (1979). Controlled and automatic human information processing: I. Detection, search, and attention. Psychological Review, 84, 1-66.

SPELKe, E. S., HIRST, W. C., \& NeISSER, U. (1976). Skills of divided attention. Cognition, 4, 215-230.

Treisman, A., \& GefFen, G. (1967). Selective attention: Perception or response? Quarterly Journal of Experimental Psychology, 19, 1-17.

(Manuscript received for publication June 5, 1984.) 